\title{
Pyomyositis of the iliacus muscle and pyogenic sacroiliitis after sacroiliac joint block
}

\section{-A case report-}

\author{
Mi Hyeon Lee ${ }^{1}$, Hyo-Jin Byon ${ }^{2}$, Hyun Jun Jung ${ }^{2}$, Young-Deog Cha², and Doo Ik Lee ${ }^{2}$ \\ ${ }^{1}$ Medical Department, Graduate School, Inha University, ${ }^{2}$ Department of Anesthesiology and Pain Medicine, Inha University \\ Hospital, Inha University College of Medicine, Incheon, Korea
}

Sacroiliac joint block can be performed for the diagnosis and treatment of sacroiliac joint dysfunction. Although sacroiliac joint block is a common procedure, complications have not been reported in detail. We report a case of iliacus pyomyositis and sacroiliac joint infection following a sacroiliac joint block. A 70-year-old female patient received sacroiliac joint blocks to relieve pelvic pain. The patient was admitted to the emergency room two days after the final sacroiliac joint block (SIJB) with the chief complaints of left pelvic pain corresponding to a visual analogue scale (VAS) score of 9 and fever. A pelvic MRI indicated a diagnosis of myositis. After 1 month of continuous antibiotic therapy, the patient's erythrocyte sedimentation rate (ESR) and C-reactive protein (CRP) level remained elevated. A ${ }^{67} \mathrm{Ga}$ SPECT/CT was done. Abnormal uptake was seen at the left sacroiliac joint (SIJ), and septic sacroiliitis was suspected. The CRP normalized to $0.29 \mathrm{mg} / \mathrm{dl}$ and the ESR decreased to $60 \mathrm{~mm} / \mathrm{hr}$, and the patient had no fever after 57 days of antibiotic therapy. She was directed for follow up at an outpatient clinic. (Korean J Anesthesiol 2013; 64: 464-468)

Key Words: Iliacus, Infection, Pyomyositis, Sacroiliac.

The sacroiliac joint (SIJ) is a synovial articulation located between the ventral wing of the ilium and the dorsal wing of the sacrum. The joint is held by three pairs of extremely strong sacroiliac ligaments [1]. At the anterior region of the SIJ, the iliopsoas and piriformis muscles are situated, and the gluteal muscle is situated at the posterior region. These surrounding ligamentous structures and muscles support the SIJ and give the joint a high degree of stability because of the constraint in its motion [2,3]. The SIJ has a small capsule and is close to the articular margins making it easy for osteoarthritis to occur [4].

In patients with low back pain, $10-25 \%$ of the primary pain occurs in the SIJ $[5,6]$. The causes of SIJ pain are very diverse and include trauma, metabolic disorders, inflammatory diseases, infections, tumors, degenerative diseases, iatrogenic conditions, referred pain, and sacroiliac joint dysfunction (SIJD) [7-9]. Although physical examinations test for motion palpation

Received: June 4, 2012. Accepted: August 1, 2012.

Corresponding author: Hyo-Jin Byon, M.D., Department of Anesthesiology and Pain Medicine, Inha University Hospital, Inha University College of Medicine, 7-206, 3-ga, Shinheung-dong, Incheon 400-711, Korea. Tel: 82-32-890-3968, Fax: 82-32-881-2476, E-mail: jinoben@hanmail.com @) This is an open-access article distributed under the terms of the Creative Commons Attribution Non-Commercial License (http:// creativecommons.org/licenses/by-nc/3.0/), which permits unrestricted non-commercial use, distribution, and reproduction in any medium, provided the original work is properly cited. 
and provocation tests can be useful in the diagnosis of SIJD, the condition is difficult to accurately diagnose. Radiological tools are ineffective for diagnosing SIJD. To date, the most effective method found for diagnosing SIJD is sacroiliac joint block (SIJB) [10]. Subsidence of pain after SIJB with steroids and local anesthetic agents provides confirmation of the diagnosis of SIJD and can simultaneously be the treatment [10]. This case deals with pyomyositis of the iliacus muscle and pyogenic sacroiliitis after SIJB.

\section{Case Report}

A 70-year-old female patient visited our pain clinic. She had a previous medical history of hypertension and tension headache for 10 years. Seven years ago, the patient had undergone L3-S1 posterolateral spinal fusion surgery after being diagnosed with spinal stenosis and spondylolisthesis. The surgery, however, led to no improvement in the patient's lumbar back pain and lumbar radiculopathy. In order to treat the continuing low back pain, pelvic pain, and spread of radicular pain to both sides of the lower leg, several epidural and caudal blocks were performed. Although the lumbar back pain improved for 7 months with treatment, the patient continued to experience pelvic pain on both sides, corresponding to a score of 9 on the visual analogue scale (VAS) ranging from $0-10(0=$ no pain, $10=$ most intense pain ever). Physical examination showed no signs of erythema, or infection. There was tenderness upon palpating both SIJs, but not on the hip joint. Patrick's and Gaenslen's tests were positive on both sides. We decided to perform SIJB for diagnostic and therapeutic purposes. We injected $0.5 \%$ mepivacaine $2 \mathrm{cc}$ and triamcinolone acetate 10 mg into the SIJs. During the SIJB, we confirmed the referred pain the patient had complained of. Two hours after the procedure, the patient's pain level decreased to a VAS score of 0 and she was discharged.

Three months later, the patient was re-admitted with a chief complaint of bilateral pelvic pain corresponding to a VAS score of 6. Physical examination showed the same results as previously. Recurrence of SIJD was suspected, and SIJB was planned. We performed SIJB on the left SIJ first to diagnose and treat the patient. After placement of non-invasive blood pressure and pulse oximetry monitors, the patient was placed on the fluoroscopy table in the prone position and both the hips and the pelvic region around the sacroiliac joints were disinfected widely with povidone iodine solution, after which we waited for 10 minutes. The physician cleaned his hands using a $62 \%$ ethanol gel, wore radiation protective gloves and disposable sterile gloves, and draped the patient. An anteroposterior fluoroscopic image of the SIJ was obtained. The fluoroscope was angled caudad, placing the posterior superior iliac spine and iliac crest to overlay the joint image, and was oblique to the contralateral side, placing the anterior and posterior aspects of the joint in line. A 23 gauge $6 \mathrm{~cm}$ disposable needle was advanced under coaxial technique and intermittent fluoroscopy until the joint was entered. After injection of $0.5 \mathrm{cc}$ of contrast media, we confirmed that the needle was positioned in the capsule by the spread of the contrast media in the SIJ. A mixture of $0.5 \%$ mepivacaine $2 \mathrm{ml}$ and triamcinolone acetonide $10 \mathrm{mg}$ was injected. Following the same procedure, SIJB was then performed on the right side. The patient complained of ordinary referred pain on both sides at the time of the contrast media injection. After the injection of the mixture of $0.5 \%$ mepivacaine $2 \mathrm{cc}$ and triamcinolone acetonide $10 \mathrm{mg}$, the pelvic pain on both sides decreased to a VAS score of 0 . The patient did not complain of any other symptoms and her blood pressure was within normal limits. She was stabilized in bed for one hour and then discharged.

The patient was admitted to the emergency room two days after the final SIJB with chief complaints of left pelvic pain corresponding to a VAS score of 9 and fever $\left(39.0^{\circ} \mathrm{C}\right)$. During the physical examination, the patient showed tenderness in the left hip. Blood pressure and heart rate were within normal limits. Laboratory tests showed the white blood cell count to be within normal limits, at $7.68 \mathrm{k} / \mu \mathrm{l}$, but the neutrophil count was increased to $93.6 \%$. Both ESR, at $35 \mathrm{~mm} / \mathrm{hr}$, and C-reactive protein (CRP), at $15 \mathrm{mg} / \mathrm{dl}$, showed an increase. There were no specific findings on chest computed tomography (CT) or abdominal and pelvic CT, with the exception of L3-S1 posterior fusion. CT showed osteophytes and erosion in both SI joints and irregularity in the joint spaces, but there were no particular changes compared to the previous CT. Following admission, the patient was started on vancomycin hydrochloride $1 \mathrm{~g}$ intravenous (IV) every 12 hours. Pelvic magnetic resonance imaging (MRI) performed 3 days after the procedure showed high signal intensity edema and enhancement in a T2weighted image at the left iliacus muscle, leading to a suspected diagnosis of myositis. There was no suspicion of infection or inflammation at the sacroiliac joint (Fig. 1). Methicillinsensitive staphylococcus aureus (MSSA) was discovered in the blood culture taken 7 days after the procedure. Following this blood culture result, the patient's antibiotic therapy was changed from vancomycin to cefazolin sodium $2 \mathrm{~g}$ IV every 8 hours. After 17 days of continuous antibiotic therapy, the patient's ESR increased to $99 \mathrm{~mm} / \mathrm{hr}$ and her CRP increased to $8.8 \mathrm{mg} / \mathrm{dl}$. Taking these findings into consideration, the antibiotics were changed to ciprofloxacin $400 \mathrm{mg}$ IV every 12 hours and rifampicin $450 \mathrm{mg}$ P.O. every 12 hours. 27 days after the procedure, the patient's left pelvic pain decreased to a VAS score of 3. However, the patient's ESR and CRP remained elevated, at $93 \mathrm{~mm} / \mathrm{hr}$ and $8.57 \mathrm{mg} / \mathrm{dl}$, respectively, and she 

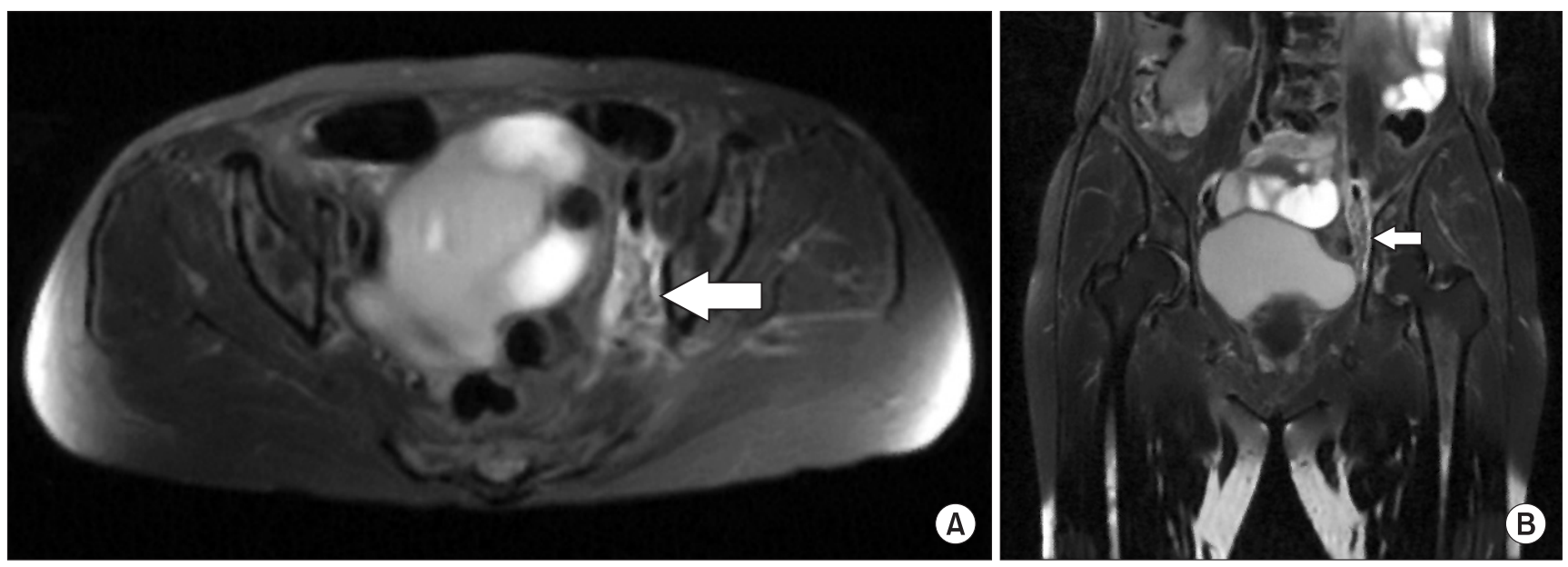

Fig. 1. This is an MRI scan of the pelvic area, showing an axial T2-weighted image (A) and coronal T2-weighted image (B). Diffuse thickening and increased signal intensity are seen in the left iliacus muscle.

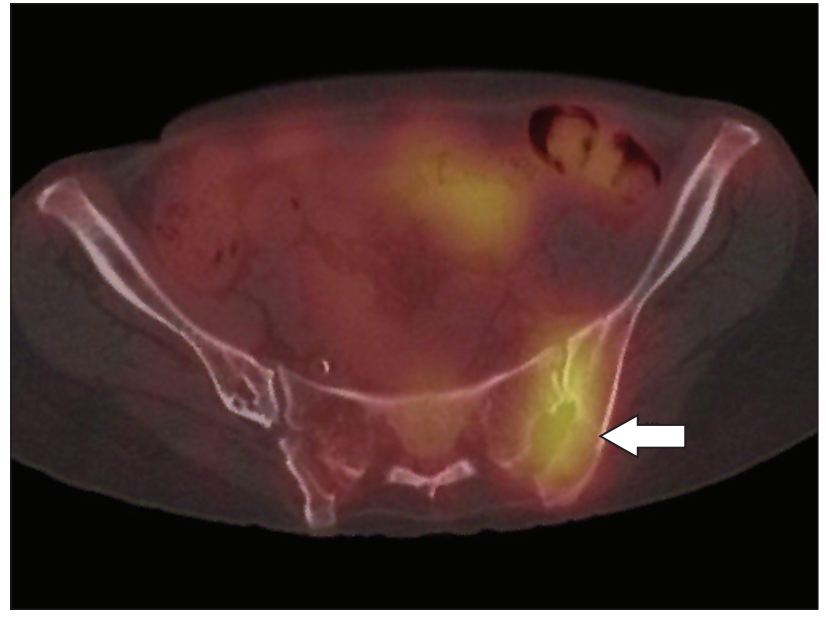

Fig. 2. This is an image from the ${ }^{67} \mathrm{Ga}$ SPECT/CT scan of the pelvic area, showing the axial view. An abnormal uptake of Gallium was observed in the left gluteal muscle and left SIJ, with bony erosion impression.

maintained a fever of at least $38.0^{\circ} \mathrm{C}$. Forty one days after the procedure, prosthetic infection at the site of the spinal fusion surgery performed 7 years ago was suspected, and ${ }^{67}$ Ga SPECT/ CT was performed. At the fusion site, ${ }^{67} \mathrm{Ga}$ SPECT/CT showed only mild uptake, which appeared to be inflammatory change. In addition, abnormal uptake was seen at the left SIJ, and septic sacroiliitis was suspected (Fig. 2). 58 days after the procedure, CRP normalized to $0.29 \mathrm{mg} / \mathrm{dl}$ and ESR decreased to $60 \mathrm{~mm} /$ $\mathrm{hr}$, and the patient had no fever. Her pain decreased to a VAS score of 2 with a fentanyl $4.2 \mathrm{mg} / 10.5 \mathrm{~cm}$ patch and oral acetaminophen. The infection was thought to be under control, so the patient was discharged and placed on antibiotics and analgesics. She was directed to follow up as an outpatient.

\section{Discussion}

This case deals with iliacus pyomyositis and pyogenic sacroiliitis following SIJB in a patient with pelvic pain. The patient experienced hip pain, tenderness, and fever 2 days after SIJB. Infection was suspected and the patient was treated with vancomycin. However, MRI of the SIJ showed nothing unusual, and iliacus myositis was suspected. Additionally, MSSA was discovered in the blood culture. Thus, the antibiotics were changed to ciprofloxacin and rifampicin. Although the patient continued to receive antibiotic treatment, her symptoms did not improve. $\mathrm{A}^{67} \mathrm{Ga}$ SPECT/CT was taken of the patient, and this revealed an infection in the SIJ. After 58 days, the patient's condition then improved with ciprofloxacin and rifampicin, and she was directed to follow up as an outpatient.

Pyogenic sacroiliitis by itself is an uncommon disease, and occurrence of iliacus pyomyositis is also a very rare case by itself $[8,11]$. Although SIJB is a common procedure, complications have not been reported in detail. There were no reports on SIJB complications from 1966 to 2006 [12]. Therefore, we feel that this case is significant because we found iliacus pyomyositis and pyogenic sacroiliitis after SIJB. This case is a valuable learning experience.

In the case discussed here, it is difficult to ascertain the cause of either iliacus pyomyositis or pyogenic sacroiliitis, and it is not possible to determine which occurred first. However, there are several possible hypotheses. First, primary pyomyositis may have occurred. Pyomyositis is a bacterial infection of the skeletal muscle. The etiology of pyomyositis is frequently classified as primary or secondary to a contiguous infection of the skin, bone, or soft tissue. Pyomyositis is most common in tropical areas, and there is a high rate of incidence in children, 
especially ages $2-5$. Individuals with a history of diabetes, alcoholism, drug abuse, HIV infection, cancer, or systemic sclerosis are at higher risk for pyomyositis. The patient has no underlying predisposing factors or conditions. The infection is believed to be a complication of transient bacteremia, which is sometimes associated with a concomitant muscle tissue structure abnormality after trauma or exercise creating a locus minoris resistentiae for implantation of bacteria. The hip and the muscles of the lower part of the body are most prone to pyomyositis, and staphylococcus aureus infection is the most common cause $[11,13]$. Due to the risk of methicillin-resistant staphylococcus aureus infection, vancomycin should be prescribed as the first choice of antibiotic therapy [14,15]. But her chronic low back and pelvic pain might induce abnormal exercise of the pelvic muscles, including the iliacus muscle, and this could be one of the causes of iliacus pyomyositis. Also, not related to the SIJB, pyogenic sacroiliitis has been reported to be a complication of primary pyomyositis [11]. Therefore, also in this case, one cannot exclude the possibility that pyomyositis and pyogenic sacroiliitis occurred due to hematogenous origin.

Second, the cause of the infection may be related to the SIJB. During the SIJB, possible contamination could have occurred due to the physician's hands, needles, drugs, or the area of the block. In this case, the physician washed his hands using $62 \%$ ethanol gel and wore disposable sterile gloves. For both SIJBs, the medications used, including mepivacaine and triamcinolone acetonide, were within the expiration date, stored according to the manufacturer's recommendations, opened immediately prior to use, and not reused. Disposable needles were used during the procedure and the tips of the needles were not bent or touched. Although all proper precautions were taken, it is not possible to completely prevent the possibility of contamination.

Third, iliacus pyomyositis and pyogenic sacroiliitis can be caused due to complex factors. Pyomyositis and pyogenic sacroiliitis may be caused by the same or different factors. After infection at the iliacus muscle or SIJ due to hematogenous origin, the infection could have moved. In all cases, steroid injection increases the possibility of infection.

We were also interested in the order of occurrence of iliacus myositis and pyogenic sacroiliitis. Although 3 days after the procedure, myositis was suspected due to the MRI image, there were no signs of infection at the SIJ. 41 days after the procedure, infection at the SIJ was suspected due to the ${ }^{67} \mathrm{Ga}$ SPECT/CT findings. For this reason, it seems more likely that pyomyositis of the iliacus muscle first occurred, followed by infection of the SIJ. However, it is also impossible to determine which occurred first due to the performance of SIJB twice in the same region and the anti-inflammatory effect of the steroid or local anesthetics.

In this case, SIJB was used for treatment or diagnostic pur- poses. Because before the procedure, there were no symptoms to indicate a particular infectious disease, laboratory or imaging tests capable of distinguishing infectious diseases were not performed. Therefore, the infectious disease could have existed prior to the SIJB and, based on the result of the physical examination, SIJD may be incorrectly diagnosed. In other words, iliacus pyomyositis could have existed before the procedure or it could have existed with SIJD. Also, it cannot be confirmed whether iliacus pyomyositis or pyogenic sacroiliitis was directly caused by SIJB.

In conclusion, although complications for SIJB are uncommon, physicians should consider the possibility of complications occurring. Therefore, during a SIJB, one must first examine the SIJ and surrounding area for infectious disease symptoms. If possible, it is important to perform laboratory or imaging tests, such as MRI. In this way, infectious diseases can be identified, which will aid in determining the cause of infectious complications. Additionally, infectious complications can occur due to SIJB, and in order to prevent this, it is important to closely monitor the progress of the patient.

\section{Acknowledgments}

Thanks to Inha University Hospital.

\section{References}

1. Dalin G, Jeffcott LB. Sacroiliac joint of the horse. 1. Gross morphology. Anat Histol Embryol 1986; 15: 80-94.

2. Pool-Goudzwaard A, Hoek van Dijke G, Mulder P, Spoor C, Snijders C, Stoeckart R. The iliolumbar ligament: its influence on stability of the sacroiliac joint. Clin Biomech (Bristol, Avon) 2003; 18: 99-105.

3. Slipman CW, Whyte WS 2nd, Chow DW, Chou L, Lenrow D, Ellen M. Sacroiliac joint syndrome. Pain Physician 2001; 4: 143-52.

4. Rooney JR. The cause and prevention of sacroiliac arthrosis in the Standardbred horse: a theoretical study. Can Vet J 1981; 22: 356-8.

5. Bernard TN Jr, Kirkaldy-Willis WH. Recognizing specific characteristics of nonspecific low back pain. Clin Orthop Relat Res 1987; 217: 266-80.

6. Fortin JD, Aprill CN, Ponthieux B, Pier J. Sacroiliac joint: pain referral maps upon applying a new injection/arthrography technique. Part II: Clinical evaluation. Spine (Phila Pa 1976) 1994; 19: 1483-9.

7. Coventry MB, Tapper EM. Pelvic instability: a consequence of removing iliac bone for grafting. J Bone Joint Surg Am 1972; 54: 83 101.

8. Coy JT 3rd, Wolf CR, Brower TD, Winter WG Jr. Pyogenic arthritis of the sacro-iliac joint. Long-term follow-up. J Bone Joint Surg Am 1976; 58: 845-9.

9. Norman GF. Sacroiliac disease and its relationship to lower abdominal pain. Am J Surg 1968; 116: 54-6.

10. McKenzie-Brown AM, Shah RV, Sehgal N, Everett CR. A systematic review of sacroiliac joint interventions. Pain Physician 2005; 8: 11525. 
11. Roca B, Torres V. Pyomyositis of the iliacus muscle complicated with septic sacroiliitis. QJM 2008; 101: 983-4.

12. Cheng J, Abdi S. Complications of joint, tendon, and muscle injections. Tech Reg Anesth Pain Manag 2007; 11: 141-7.

13. Lorenz U, Abele-Horn M, Bussen D, Thiede A. Severe pyomyositis caused by Panton-Valentine leucocidin-positive methicillin- sensitive Staphylococcus aureus complicating a pilonidal cyst. Langenbecks Arch Surg 2007; 392: 761-5.

14. Bickels J, Ben-Sira L, Kessler A, Wientroub S. Primary pyomyositis. J Bone Joint Surg Am 2002; 84-A: 2277-86.

15. Crum-Cianflone NF. Bacterial, fungal, parasitic, and viral myositis. Clin Microbiol Rev 2008; 21: 473-94. 